\title{
Mejorando la gestion forestal a base de integrar datos laser y rodales dinámicos basados en optimización espacial
}

\author{
Improving forest management planning \\ by means of airborne laser scanning and dynamic \\ treatment units based on spatial optimization
}

\author{
Pascual, A. ${ }^{1}$
}

${ }^{1}$ University of Eastern Finland, School of Forest Sciences, P.O. Box 111, FI-80101 joensuu, Finland. 


\title{
Resumen
}

El uso de sensores laser aerotransportados (ALS) ha mejorado el inventario forestal en las últimas décadas debido a la capacidad del láser de describir la estructura del bosque en tres dimensiones. Esta investigación se centra en la integración del inventario forestal basado en ALS dentro de la planificación para crear rodales dinámicos (DTUs). En este enfoque, las unidades de gestión no son fijas ni predefinidas. Son temporales y se forman mediante la agregación de unidades de pequeña escala. Los objetivos de la planificación y los modelos de dinámica forestal son los vectores de esa agregación. La investigación se realizó en dos bosques en Castilla y León (España) en donde se tomaron observaciones de campo y datos ALS para estimar las características del bosque. Esta tesis incluye cuatro manuscritos sobre la implementación de los rodales dinámicos, las consecuencias de utilizar distintas unidades de inventario (FIU) y el impacto de los errores de posicionamiento. Los modelos de planficación incluyeron objetivos espaciales y no espaciales. Los métodos heurísticos de optimización fueron eficaces a la hora de solventar el problema de combinatoria. Los resultados destacan el buen rendimiento de las formas irregular de FIU generadas con segmentación. La optimización espacial mejoró la configuración espacial de las soluciones y con un coste bajo comparado con las soluciones no espaciales. El uso de objectivos espaciales y funciones de coste mejoró a su vez la agregación. Esta tesis muestra como la combinación de datos ALS y la optimización espacial contribuyen al desarrollo de métodos contemporáneos de planificiación.

Palabras clave: $\operatorname{xxxxxxxxxxx}$

\begin{abstract}
The use of airborne laser scanning (ALS) has enhanced forest inventory during the last decades due to the increasing capability of lasers to describe the three-dimensional structure of forests. This research focuses on the integration of ALS-based forest inventory into forest planning when the aim is to create dynamic treatment units (DTU). In this approach, the management units are not fixed and predefined. They are temporary and formed by aggregating fine-grained inventory units. Management objectives and forest dynamics are the drivers of that aggregation process. The research was conducted in two pine forests in Castilla y León (Spain) in which ground and ALS data were collected to estimate forest attributes. This $\mathrm{PhD}$ thesis reviews four manuscripts concerning the implementation of DTU, the implications of using alternative forest inventory units (FIU) in two types of problem formulations and the impact of plot positioning errors on the whole planning process. The forest management plans included spatial and non-spatial objective variables. Heuristic-based optimization was effective at solving the spatial combinatorial problems. The findings of this work highlight the good performance of irregular types of FIU derived with segmentation techniques. The use of spatial optimization improved the spatial layout of forest plans at a minor cost compared to non-spatial formulations. The use of spatial goals and spatially explicit harvest cost functions enhanced the aggregation of FIUs. This PhD shows how the combination of ALS-based methods, widely used in forestry practice, and spatial optimization contribute to the development of modern forest planning methods.
\end{abstract}

Keywords: $\operatorname{xxxxxxxxxxxxxx}$ 


\section{Introducción}

El escaneado LiDAR es una de las herramientas más potentes para describir la estructura de los ecosistemas forestales gracias nubes de puntos georreferenciadas (Vauhkonen et al., 2014). La capacidad del láser de atravesar el dosel de copas permita derivar la estructura tridimensional de la vegetación, permitiendo relacionar a su vez esta información con datos de campo también georreferenciados (Maltamo et al., 2009). El resultado son capas de información espacialmente continuas (Coops et al., 2004), como el modelo digital del terreno (DTM) o el modelo de altura de vegetación (CHM), además de las nubes de puntos. Con estas capas se puede mejorar la resolución espacial de las unidades de inventario forestal. La clusterización de pixeles del CHM se puede realizar con algoritmos de segmentación o agrupación que permitan generar unidades de inventario homogéneas y que se ajusten tanto a la distribución de la vegetación como a las irregularidades del terreno (Fig. 1). Estos algoritmos son más complejos que la simple utilización de polígonos regulares, pero permiten corregir el efecto borde (Hyvönen et al., 2005) y suponen una primera clusterización de los píxeles que forman el CHM.
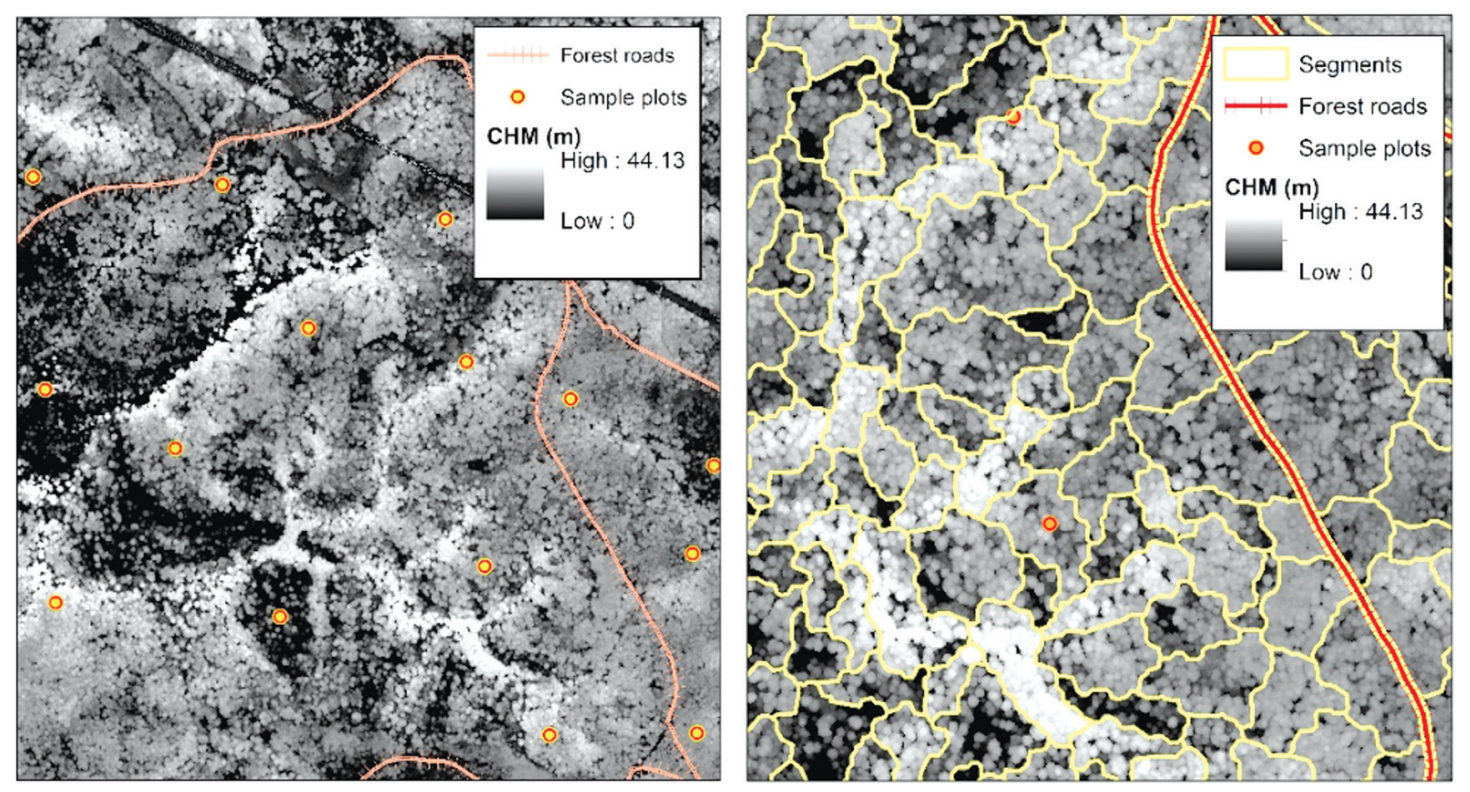

Figura 1. Izquierda: Se muestra la zona de estudio utilizando el modelo digital de altura de vegetación (CHM), las parcelas de inventario forestal y las pistas forestales. Derecha: Muestra de los segmentos utilizados en la tesis.

Existen dos métodos de trabajo para estimar las características de los ecosistemas forestales utilizando datos laser: los métodos de masa y la individualización de árboles. En los métodos de masa, se utilizan estadísticas derivadas de la 
nube de puntos como la altura de los retornos laser o la fracción de retornos sobre un determinado valor de altura, para estimar las características forestales medidas en las parcelas de campo (Næsset 2002). La detección de árboles desde la nube de puntos es el otro método de referencia en el cual la densidad de la nube de puntos y la complejidad del ecosistema forestal juegan un papel limitante (Kaartinen et al., 2012). Los métodos de masa en cambio son más fáciles de aplicar dado que la posición de los árboles dentro de las parcelas no es necesaria, basta con la medición del centro de la parcela.

La ventaja de los datos LiDAR para su aplicación en inventario forestal es clara. Trasladar esas mejoras a la toma de decisiones en planificación forestal es el siguiente paso. El objeto de la planificación forestal es proponer diferentes alternativas de gestión de los ecosistemas forestales a fin de cumplir los objetivos marcados. En el caso de la planificación forestal tradicional, las decisiones de cuándo, dónde y cómo realizar prescripciones selvícolas ha marcado la estrategia de la planificación, siempre con el objetivo de producir bienes de consumo de manera sostenible (McDill 2014). El uso de modelos de crecimiento y simuladores es posible generar alternativas de manejo para cada unas de las unidades de inventario forestal, bien sean píxeles o segmentos. Encontrar la combinación de alternativas óptimas para satisfacer los objetivos es un proceso de optimización de recursos. Con el uso de unidades de inventario de baja escala, el número de combinaciones se incrementa sustancialmente y es necesario recurrir a soluciones heurísticas en optimización espacial (Bettinger et al., 2003; Pukkala and Kurttila 2005). Las técnicas heurísticas, que descomponen el problema para acortar el proceso de optimización, son indicadas cuando el problema de combinatoria es muy complejo (Pukkala et al., 2009). Los problemas espaciales que se formularon en esta tesis es la generación de rodales dinámicos, un ejemplo de integración de datos LiDAR para la toma de decisiones que es muy necesario en el sector forestal.

Las unidades de gestión forestal son divisiones temporales y homogéneas del ecosistema forestal aunque, en la práctica, se tiende a preservar los límites establecidos de los rodales a pesar de su marcado temporal. Reconocer las unidades de gestión como unidades estancas obvia el efecto de la dinámica forestal del ecosistema y limita la integración de nuevos objetivos de gestión (de-Miguel et $a l ., 2013)$. Mediante técnicas de optimización especial y modelos de dinámica forestal es posible generar unidades de gestión a lo largo de un plan de gestión (Pukkala et al., 2014), de tal forma que las unidades cambien con el tiempo en base a los nuevos objetivos y las decisiones de anteriores periodos de gestión. Este concepto se denomina rodales dinámicos y es la base de esta tesis doctoral: cómo combinar datos láser, modelos de crecimiento y optimización para proponer un nuevo de método de planificación forestal. En el siguiente diagrama se ilustra el flujo de trabajo (Fig. 2). 


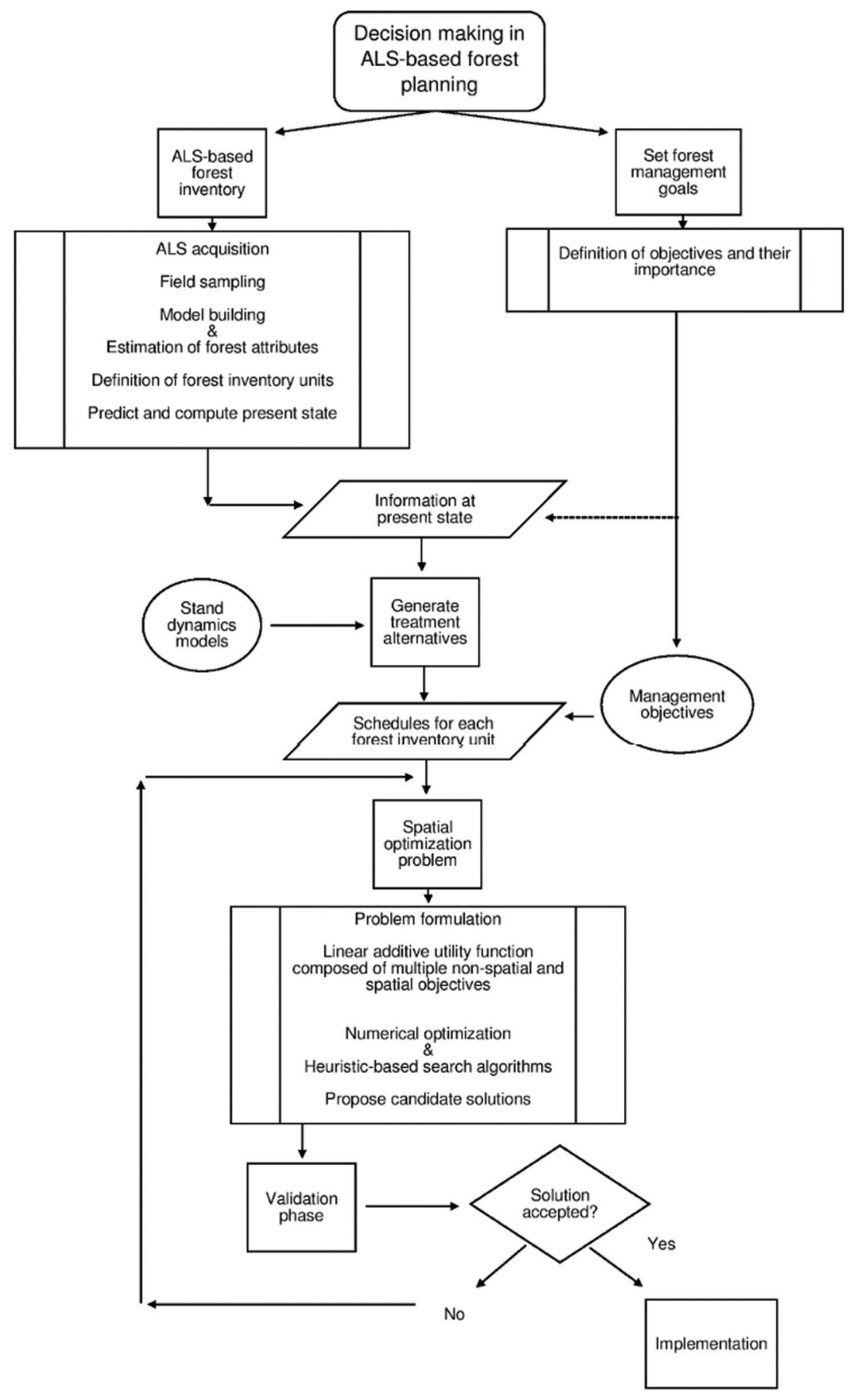

Figura 2. Proceso de integración del inventario forestal basado en datos laser en la toma de decisiones en planificación forestal. 


\section{Resultados}

El uso de segmentos como unidades de inventario mejoro la eficacia de los planes de gestión orientados a la maximización de la producción (cortas periódicas y volumen en pie a final del plan). La escala de los segmentos juega un papel importante dado que a medida que las unidades de inventario son más pequeñas, más posibilidades de combinatoria existen y así se consiguió mejorar la optimización en base a segmentos. La adición de objetivos espaciales, orientados a agregar más las unidades de inventario, se tradujo en una pérdida de eficiencia cuando se comparan los valores con las formulaciones no espaciales (Tab. 1). En vista de los resultados, el uso de pixeles de 0.05 ha en formulaciones no espaciales no es implementable (Pascual et al., 2016; Pascual et al., 2018). Sin embargo, la adición de objetivos espaciales mejoró notablemente la agregación de píxeles y segmentos (Fig. 3), a la vez que redujo la dispersión de las soluciones: agregaciones de pequeño tamaño que distan de los rodales grandes y compactos (Pascual et al., 2018).

Tabla 1. Producción de madera (cortas y volumen a final del periodo de gestión) para las tres unidades de inventario (FIU) testadas en la tesis. Se muestra también la duración de la optimización.

\begin{tabular}{ccccc}
\hline \multirow{2}{*}{ FIU } & \multicolumn{2}{c}{ Problema no espacial } & \multicolumn{2}{c}{ Problema espacial } \\
\cline { 2 - 5 } & $\begin{array}{c}\text { Producción } \\
\left(\mathrm{m}^{3} \mathrm{ha}^{-1}\right)\end{array}$ & $\begin{array}{c}\text { Solución } \\
(\mathrm{s})\end{array}$ & $\begin{array}{c}\text { Production } \\
\left(\mathrm{m}^{3} \mathrm{ha}^{-1}\right)\end{array}$ & $\begin{array}{c}\text { Solución } \\
(\mathrm{s})\end{array}$ \\
\hline Segmentos grandes & 154.1 & 91 & 145.4 & 225 \\
Segmentos pequeños & 159.1 & 367 & 149.0 & 894 \\
Píxeles & 153.6 & 3117 & 142.2 & 8636 \\
\hline
\end{tabular}

En la tesis también se mostró cómo el uso de funciones de coste espacialmente dependientes y estimadas con datos láser ayudó a mejorar el rendimiento económico de la planificación. La distribución espacial de los rodales dinámicos se delimitó a aquellas áreas en las que el aprovechamiento era económicamente rentable y esto a su vez, fue una vía indirecta de agregación de unidades de inventario. Maximizar la producción forestal sin atender al rendimiento económico de las decisiones puede ocasionar una pérdida de rendimiento importante como se demostró en el artículo IV de esta tesis (Pascual et al., 2018). En la misma línea, los errores de posicionamiento también supusieron una pérdida menor de rendimiento debido a errores en la toma de decisiones ocasionados por alterar la relación entre datos de campos y estadísticas LiDAR. El papel de la ecosistema forestal y el diseño experimental pueden en parte explicar la reducida magnitud del impacto (Pascual et al., 2018). 


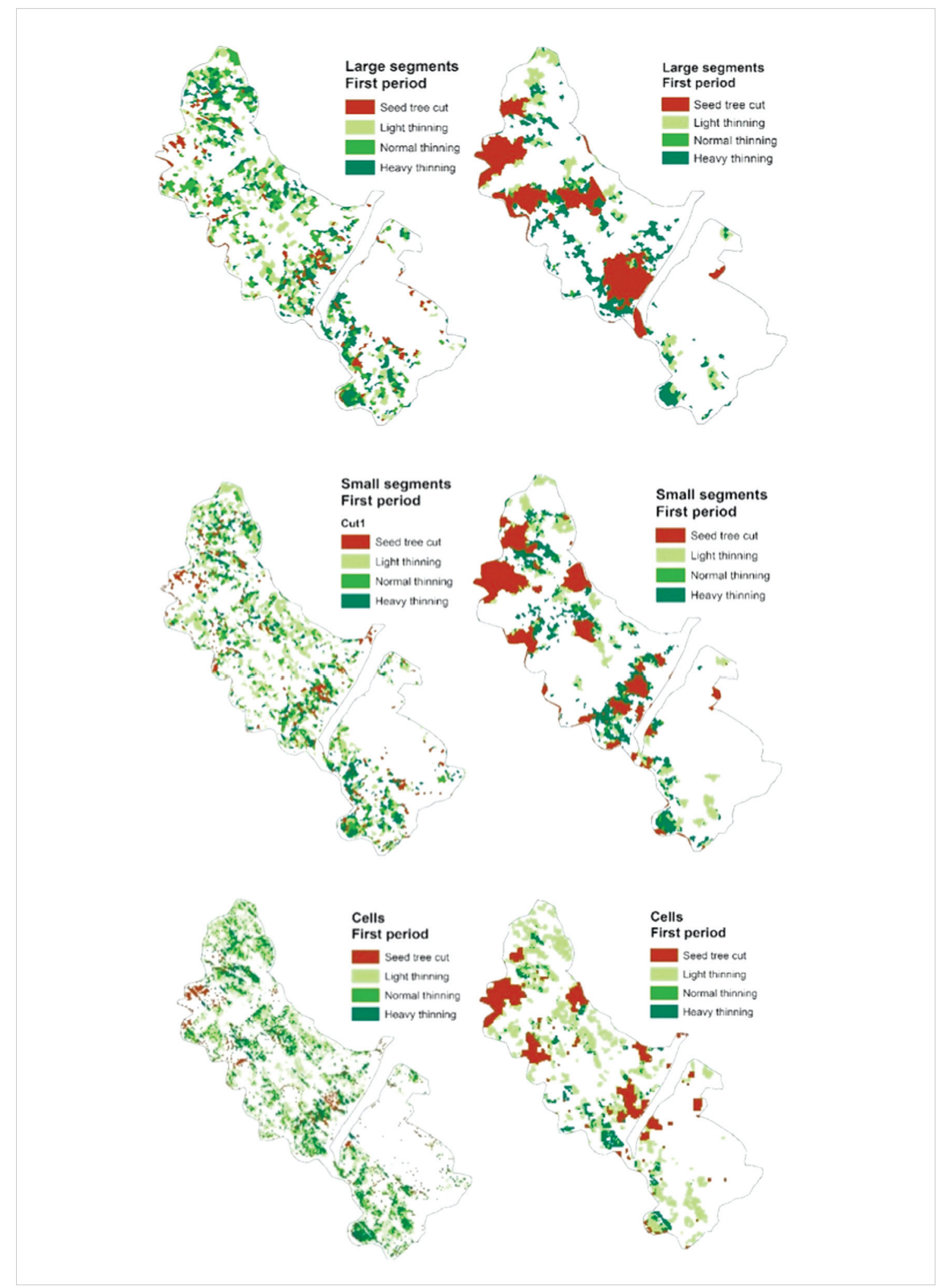

Figura 3. Rodales dinámicos aplicando formulación no espacial (izquierda) y espacial (derecha) para las tres unidades de inventario testadas: segmentos grandes, pequeños y píxeles de baja escala (0.05 ha). 


\section{Discusión y conclusiones}

Los cuatro artículos que componen la tesis doctoral muestran cómo extender la precisión del inventario forestal con datos LiDAR hacia la toma de decisiones en planificación forestal. Las unidades de inventario definas mediante segmentación con datos laser y la estimación de las características del ecosistema forestal con esta metodología permiten ambas una mejor evaluación de los recursos forestales. Si bien los avances en inventario forestal ya están plenamente consolidados a nivel operacional, la innovación en las metodologías de planificación forestal es inexistentes. En este sentido, la metodología de rodales dinámicos supone un cambio profundo en cómo ordenar un ecosistema forestal.

Los cuatro artículos de esta tesis doctoral incluyen objetivos económicos, productivos y espaciales a la hora de generar rodales dinámicos. Es importante reconocer el componente multi-objetivo de las soluciones y para ello los métodos de toma de decisiones deben ser flexibles y dinámicos. Y los rodales dinámicos lo son. La vigencia de los planes de ordenación de nuestros ecosistemas forestal en España es de varias decenas de años. Mantener las unidades de gestión tal y como se definieron en los primeros planes de ordenación es la tónica dominante en el sector. Sin embargo, las actuaciones selvícolas, la dinámica forestal o simplemente un cambio de objetivos en la planificación puede hacer que la delineación de rodales existentes no sea óptima. Es buen momento para consolidar la aplicación de la tecnología LiDAR en el sector y seguir avanzando en mejorar el siguiente proceso: la toma de decisiones.

Implementar la metodología de rodales dinámicos en España es posible dado el libre acceso a datos LiDAR de baja resolución y al amplio repositorio de modelos de dinámica forestal desarrollado en las últimas décadas en base a la información del Inventario Forestal Nacional. Los datos LiDAR y las parcelas de campo definen el momento actual del inventario, que se actualiza con los modelos y las decisiones sobre cada una de las unidades de inventario. En este sentido, el uso de algoritmos heurísticos va a ser importante para optimizar la creación de rodales dinámicos usando unidades de baja escala. Los métodos basados en soluciones exactas, como la programación lineal, no son efectivos cuando son miles las unidades de inventario involucradas. Sin embargo, sí que pueden ser un enfoque válido usando, por ejemplo, los rodales actuales dentro de formulaciones que incluyan objetivos adicionales al ya clásico de maximización de la producción forestal con cortas periódicas.

El uso combinado de datos LiDAR, modelización, parcelas permanentes y optimización espacial van a permitir actualizar y modernizar la planificación forestal en España. Ese has sido el objetivo primordial de esta tesis doctoral.

\section{Bibliografía}

Bettinger, P.; Johnson, D.L.; Johnson, K.N.; 2003. Spatial forest plan development with eco- 
logical and economic goals. Ecol. Modell. 169, 215-236. https://doi.org/10.1016/S03 04-3800(03)00271-0

Coops, N.C.; Wulder, M.A.; Culvenor, D.S.; St-Onge B.; 2004. Comparison of forest attributes extracted from fine spatial resolution multispectral and lidar data. Can. J. Remote. Sens. 30, 855-866. https://doi.org/10.5589/m04-045

De-Miguel, S.; Pukkala, T.; Pasalodos, J.; 2013. Dynamic treatment units: flexible and adaptive forest management and planning by combining spatial optimization methods and LiDAR. Cuadernos de la Sociedad Española de Ciencias Forestales, 37:49-54. [In Spanish]

Hyvönen, P.; Pekkarinen, A.; Tuominen, S.; 2005. Segment-level stand inventory for forest management. Scand. J. Forest. Res. 20, 75-84. https://doi.org/10.1080/028275805100 08220

Kaartinen, H.; Hyyppä, J.; Yu, X.; et al., 2012. An international comparison of individual tree detection and extraction using airborne laser scanning. Remote Sens. 4, 950-974. https://doi.org/10.3390/rs4040950

Maltamo, M.; Peuhkurinen, J.; Malinen, J.; Vauhkonen, J.; Tokola, T.; 2009. Predicting Tree Attributes and Quality Characteristics of Scots Pine Using Airborne Laser Scanning Data. Silva Fenn. 43, 507-521. https://doi.org/10.14214/sf.203

McDill, E.; 2014. An overview of forest management planning and information management. In: Borges, J.; et al., (eds), The management of industrial forest plantations: theoretical foundations and applications; Manag. For. Ecosyst. 33. Springer, Dordrecht. https://doi.org/10.1007/978-94-017-8899-1_2

Næsset, E.; 2002. Predicting forest stand characteristics with airborne scanning laser using a practical two-stage procedure and field data. Remote. Sens. Environ. 80, 88-99. https://doi.org/10.1016/S0034-4257(01)00290-5

Pascual, A.; Pukkala, T.; Rodríguez, F.; de-Miguel, S.; 2016. Using Spatial Optimization to Create Dynamic Harvest Blocks from LiDAR-Based Small Interpretation Units. Forests 7(10), 220. https://doi.org/10.3390/f7100220

Pascual, A.; Pukkala, T.; de-Miguel, S.; 2018. Effects of plot positioning errors on the optimality of harvest prescriptions in spatial forest planning based on ALS data. Forests 9 (7), 371. https://doi.org/10.3390/f9070371

Pascual, A.; Pukkala, T.; de-Miguel, S.; Pesonen, A.; Packalen, P.; 2018. Influence of size and shape of forest inventory units on the layout of harvest blocks in numerical forest planning. Eur. J. For. Res. 138, 112-123. https://doi.org/10.1007/s10342-018-1157-5

Pascual, A.; Pukkala, T.; de-Miguel, S.; Pesonen, A.; Packalen, P.; 2018 Influence of timber harvesting costs on the layout of cuttings and economic return in forest planning based on dynamic treatment units. Forest Syst. 27, 1. https://doi.org/10.5424/fs/2018271-11 897

Pukkala, T.; Kurttila, M.; 2005. Examining the performance of six heuristic optimisation techniques in different forest planning problems. Silva Fenn. 39, 67-80. https://doi.org/ $10.14214 /$ sf.396

Vauhkonen, J.; Maltamo, M.; McRoberts, R.E.; Næsset, E.; 2014. Introduction to Forestry Applications of Airborne Laser Scanning. In: M. Maltamo et al., (eds.), Forestry Applications of Airborne Laser Scanning: Concepts and Case Studies. Manag. For. Ecosyst. 27. Springer. The Netherlands. https://doi.org/10.1007/978-94-017-8663-8 


\section{Datos de la tesis}

Titulo:Improving forest management planning by means of airborne laser scanning and dynamic treatment units based on spatial optimization / Mejorando la gestion forestal a base de integrar datos laser y rodales dinámicos basados en optimización espacial.

Director de la tesis: Timo Pukkala.

Universidad: University of Eastern Finland

Fecha de defensa: 07/09/2018

Calificación: Excelente.

Ubicación en repositorio: https://dissertationesforestales.fi/article/10020

\section{Artículos que conforman la tesis:}

Pascual, A.; Pukkala, T.; Rodríguez, F.; de-Miguel, S.; 2016. Using Spatial Optimization to Create Dynamic Harvest Blocks from LiDAR-Based Small Interpretation Units. Forests 7(10), 220.

Pascual, A.; Pukkala, T.; de-Miguel, S.; 2018. Effects of plot positioning errors on the optimality of harvest prescriptions in spatial forest planning based on ALS data. Forests 9(7), 371.

Pascual, A.; Pukkala, T.; de-Miguel, S.; Pesonen, A.; Packalen, P.; 2018. Influence of size and shape of forest inventory units on the layout of harvest blocks in numerical forest planning. European Journal of Forest Research 138(1), 111-123.

Pascual, A.; Pukkala, T.; de-Miguel, S.; Pesonen, A.; Packalen, P.; 2018. Influence of timber harvesting costs on the layout of cuttings and economic return in forest planning based on dynamic treatment units. Forest Systems 27:1. 